\title{
Torus Actions Determined by Simple Polytopes
}

\author{
Victor M. Buchstaber and Taras E. Panov
}

\begin{abstract}
An $n$-dimensional polytope $P^{n}$ is called simple if exactly $n$ codimension-one faces meet at each vertex. The lattice of faces of a simple polytope $P^{n}$ with $m$ codimension-one faces defines an arrangement of coordinate subspaces in $\mathbb{C}^{m}$. The group $\mathbb{R}^{m-n}$ acts on the complement of this arrangement by dilations. The corresponding quotient is a smooth manifold $\mathcal{Z}_{P}$ invested with a canonical action of the compact torus $T^{m}$ with the orbit space $P^{n}$. For each smooth projective toric variety $M^{2 n}$ defined by a simple polytope $P^{n}$ with the given lattice of faces there exists a subgroup $T^{m-n} \subset T^{m}$ acting freely on $\mathcal{Z}_{P}$ such that $\mathcal{Z}_{P} / T^{m-n}=M^{2 n}$. We calculate the cohomology ring of $\mathcal{Z}_{P}$ and show that it is isomorphic to the cohomology of the Stanley-Reisner ring of $P^{n}$ regarded as a module over the polynomial ring. In this way the cohomology of $\mathcal{Z}_{P}$ acquires a bigraded algebra structure, and the additional grading allows to catch combinatorial invariants of the polytope. At the same time this gives an example of explicit calculation of the cohomology ring for the complement of a subspace arrangement defined by simple polytope, which is of independent interest.
\end{abstract}

\section{Introduction}

A convex $n$-dimensional polytope $P^{n}$ is called simple if exactly $n$ codimensionone faces meet at each vertex. Such polytopes are generic points in the variety of all $n$-dimensional convex polytopes. One can associate to each simple polytope $P^{n}$ a smooth $(m+n)$-dimensional manifold $\mathcal{Z}_{P}$ with canonical action of the torus $T^{m}$ on it; here $m$ is the number of codimension-one faces of $P^{n}$.

A number of manifolds playing an important role in different aspects of topology, algebraic and symplectic geometry are quotients $\mathcal{Z}_{P} / T^{k}$ for the action of some subgroup $T^{k} \subset T^{m}$. The most well-known class of such manifolds are the (smooth, projective) toric varieties in algebraic geometry. From the viewpoint of our approach, these toric varieties (or toric manifolds) correspond to those simple polytopes $P^{n}$ for which there exists a torus subgroup in $T^{m}$ of maximal possible rank $m-n$ that acts freely on $\mathcal{Z}_{P}$. Thus, all toric manifolds can be obtained as quotients of $\mathcal{Z}_{P}$ by a torus of above type.

1991 Mathematics Subject Classification. Primary 57R19, 57S25; Secondary 14M25, 52B05.

Partially supported by the Russian Foundation for Fundamental Research, grant no. 99-0100090, and INTAS, grant no. 96-0770. 
The manifolds $\mathcal{Z}_{P}$ were firstly introduced in $[\mathbf{D J}]$ via certain equivalence relation $\sim$ as $\mathcal{Z}_{P}=T^{m} \times P^{n} / \sim$ (see Definition 1.2). We propose another approach to defining $\mathcal{Z}_{P}$ based on a construction from the algebraic geometry of toric varieties. This construction was initially used in $[\mathbf{B a}]$ (see also $[\mathbf{A u}],[\mathbf{C o}]$ ) for definition of toric manifolds. Namely, the combinatorial structure of $P^{n}$ defines an algebraic set $U\left(P^{n}\right) \subset \mathbb{C}^{m}$ (the complement of a certain arrangement of coordinate subspaces, see Definition 2.1) with action of the group $\left(\mathbb{C}^{*}\right)^{m}$ on it. Toric manifolds appear when one can find a subgroup $D \subset\left(\mathbb{C}^{*}\right)^{m}$ isomorphic to $\left(\mathbb{C}^{*}\right)^{m-n}$ that acts freely on $U\left(P^{n}\right)$. However, it turns out that it is always possible to find a subgroup $R \subset\left(\mathbb{C}^{*}\right)^{m}$ isomorphic to $\mathbb{R}^{m-n}$ that acts on $U\left(P^{n}\right)$ freely. Then, for each subgroup $R$ of such kind the corresponding quotient $U\left(P^{n}\right) / R$ is homeomorphic to $\mathcal{Z}_{P}$.

One of our main goals here is to study relationships between the combinatorial structure of simple polytopes and the topology of the above manifolds. One aspect of this relation is the existence of a certain bigraded complex calculating the cohomology of $\mathcal{Z}_{P}$. This bigraded complex arises from the interesting geometric structure on $\mathcal{Z}_{P}$, which we call bigraded cell structure. This structure is defined by the torus action and the combinatorics of polytope. Thus, it seems to us that the above manifolds defined by simple polytopes could be also used as a powerful combinatorial tool.

Part of results of this article were announced in [BP1].

The authors express special thanks to Nigel Ray, since the approach to studying the manifolds $\mathcal{Z}_{P}$ described here was partly formed during the work on the article $[\mathbf{B R}]$.

\section{Manifolds defined by simple polytopes}

We start with reviewing some basis combinatorial objects associated with simple polytopes. The good references here are $[\mathbf{B r}]$ and $[\mathbf{Z i}]$.

For any simple $P^{n}$, let $f_{i}$ denote the number of faces of codimension $(i+1)$, $0 \leq i \leq n-1$. The integer vector $\left(f_{0}, \ldots, f_{n-1}\right)$ is called the $f$-vector of $P^{n}$. It is convenient to set $f_{-1}=1$. We will also consider the another integral vector $\left(h_{0}, \ldots, h_{n}\right)$ called $h$-vector of $P^{n}$, where $h_{i}$ are retrieved from the formula

$$
h_{0} t^{n}+\ldots+h_{n-1} t+h_{n}=(t-1)^{n}+f_{0}(t-1)^{n-1}+\ldots+f_{n-1},
$$

that is,

$$
\sum_{i=0}^{n} h_{i} t^{n-i}=\sum_{i=0}^{n} f_{i-1}(t-1)^{n-i}
$$

This implies that

$$
h_{k}=\sum_{i=0}^{k}(-1)^{k-i}\left(\begin{array}{l}
n-i \\
k-i
\end{array}\right) f_{i-1} .
$$

Now let $\mathcal{F}=\left(F_{1}^{n-1}, \ldots, F_{m}^{n-1}\right)$ be the set of all codimension-one faces of $P^{n}$, so $m=f_{0}$. We fix a commutative ring $\mathbf{k}$, which we refer to the as ground ring.

Definition 1.1. The face ring (or the Stanley-Reisner ring) $\mathbf{k}\left(P^{n}\right)$ is defined to be the ring $\mathbf{k}\left[v_{1}, \ldots, v_{m}\right] / I$, where

$$
I=\left(v_{i_{1}} \ldots v_{i_{s}}: \quad i_{1}<i_{2}<\ldots<i_{s}, \quad F_{i_{1}} \cap F_{i_{2}} \cap \cdots \cap F_{i_{s}}=\varnothing\right) .
$$


Thus, the face ring is a quotient ring of polynomial ring by an ideal generated by some square free monomials of degree $\geq 2$. We make $\mathbf{k}(K)$ a graded ring by setting $\operatorname{deg} v_{i}=2, i=1, \ldots, m$.

For any simple polytope $P^{n}$ one can define $(n-1)$-dimensional simplicial complex $K_{P}$ dual to the boundary $\partial P^{n}$. Originally, face ring was defined by Stanley $[\mathbf{S t 2}]$ for simplicial complexes. In our case Stanley's face ring $\mathbf{k}\left(K_{P}\right)$ coincides with $\mathbf{k}\left(P^{n}\right)$.

Below for any simple $P^{n}$ with $m$ codimension-one faces we define, following $[\mathbf{D J}]$, two topological spaces $\mathcal{Z}_{P}$ and $B_{T} P$.

Set the standard basis $\left\{e_{1}, \ldots, e_{m}\right\}$ in $\mathbb{Z}^{m}$, and define canonical coordinate subgroups $T_{i_{1}, \ldots i_{k}}^{k} \subset T^{m}$ as tori corresponding to the sublattices spanned in $\mathbb{Z}^{m}$ by $e_{i_{1}}, \ldots, e_{i_{k}}$.

Definition 1.2 . The space $\mathcal{Z}_{P}$ associated with simple polytope $P^{n}$ is $\mathcal{Z}_{P}=$ $\left(T^{m} \times P^{n}\right) / \sim$, where the equivalence relation $\sim$ is defined as follows: $\left(g_{1}, p\right) \sim$ $\left(g_{2}, q\right) \Longleftrightarrow p=q$ and $g_{1} g_{2}^{-1} \in T_{i_{1}, \ldots, i_{k}}^{k}$. Here $\left\{i_{1}, \ldots, i_{k}\right\}$ is the set of indices of all codimension-one faces containing the point $p \in P^{n}$, that is, $p \in F_{i_{1}} \cap \cdots \cap F_{i_{k}}$.

Note that $\operatorname{dim} \mathcal{Z}_{P}=m+n$. The torus $T^{m}$ acts on $\mathcal{Z}_{P}$ with orbit space $P^{n}$. This action is free over the interior of $P^{n}$ and has fixed points corresponding to vertices of $P^{n}$. It was mentioned above that there are other well-known in algebraic geometry examples of manifolds with torus action and orbit space a simple polytope. These are the toric varieties $[\mathbf{D a}],[\mathbf{F u}]$ (actually, we consider only smooth projective toric varieties). The space $\mathcal{Z}_{P}$ is related to this as follows: for any smooth toric variety $M^{2 n}$ over $P^{n}$ the orbit map $\mathcal{Z}_{P} \rightarrow P^{n}$ decomposes as $\mathcal{Z}_{P} \rightarrow M^{2 n} \rightarrow P^{n}$, where $\mathcal{Z}_{P} \rightarrow M^{2 n}$ is a principal $T^{m-n}$-bundle, and $M^{2 n} \rightarrow P^{n}$ is the orbit map for $M^{2 n}$. We will review this connection with more details later.

EXAMPLE 1.3. Let $P^{n}=\Delta^{n}$ (an $n$-dimensional simplex). Then $m=n+1$, and it is easy to check that $\mathcal{Z}_{P}=\left(T^{n+1} \times \Delta^{n}\right) / \sim \cong S^{2 n+1}$.

Using the action of $T^{m}$ on $\mathcal{Z}_{P}$, define the homotopy quotient (the Borel construction)

$$
B_{T} P=E T^{m} \times_{T^{m}} \mathcal{Z}_{P},
$$

where $E T^{m}$ is the contractible space of universal $T^{m}$-bundle over $B T^{m}=\left(\mathbb{C} P^{\infty}\right)^{m}$. It is clear that the homotopy type of $B_{T} P$ is defined by the simple polytope $P^{n}$.

Let $I^{q}$ be the standard $q$-dimensional cube in $\mathbb{R}^{q}$ :

$$
I^{q}=\left\{\left(y_{1}, \ldots, y_{q}\right) \in \mathbb{R}^{q}: 0 \leq y_{i} \leq 1, i=1, \ldots, q\right\} .
$$

A cubical complex is a topological space represented as the union of homeomorphic images of standard cubes in such a way that the intersection of any two cubes is a face of each.

LEMMA 1.4. Any simple polytope $P^{n}$ has a natural structure of cubical complex $\mathcal{C}$, which has $s=f_{n-1} n$-dimensional cubes $I_{v}^{n}$ indexed by the vertices $v \in P^{n}$ and $1+f_{0}+f_{1}+\ldots+f_{n-1}$ vertices. Moreover there is a natural embedding $i_{P}$ of $\mathcal{C}$ into the boundary complex of standard $m$-dimensional cube $I^{m}$.

Proof. Let us take a point in the interior of each face of $P^{n}$ (we also take all vertices and a point in the interior of the polytope). The resulting set $\mathcal{S}$ of $1+f_{0}+f_{1}+\ldots+f_{n-1}$ points is said to be the vertex set of the cubical complex 

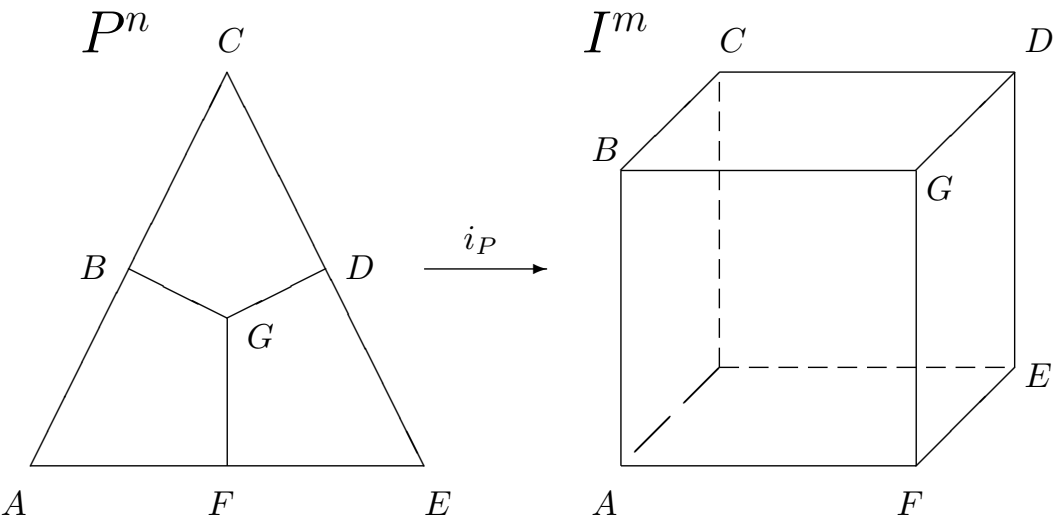

Figure 1. The embedding $i_{p}: P^{n} \rightarrow I^{m}$ for $n=2, m=3$.

$\mathcal{C}$. Now let us construct the embedding $\mathcal{C} \hookrightarrow I^{m}$. We map the point of $\mathcal{S}$ that lies inside the face $F^{n-k}=F_{i_{1}}^{n-1} \cap \cdots \cap F_{i_{k}}^{n-1}, k \geq 1$ to the vertex of the cube $I^{m}$ whose $y_{i_{1}}, \ldots, y_{i_{k}}$ coordinates are zero, while other coordinates are 1 . The point of $\mathcal{S}$ in the interior of $P^{n}$ is then mapped to the vertex of $I^{m}$ with coordinates $(1, \ldots, 1)$. Let us consider the simplicial subdivision $\mathcal{K}$ of the polytope $P^{n}$ that is constructed as the cone over the barycentric subdivision of simplicial complex $K^{n-1}$ dual to the boundary of $P^{n}$. The vertex set of the simplicial complex $\mathcal{K}$ is our set $\mathcal{S}$, and for any vertex $v$ of $P^{n}$ one can find a subcomplex $\mathcal{K}_{v} \subset \mathcal{K}$ (the cone over the barycentric subdivision of the $(n-1)$-simplex in $K^{n-1}$ corresponding to $\left.v\right)$ that simplicially subdivides the cube $I_{v}^{n}$. Now we can extend the map $\mathcal{S} \hookrightarrow I^{m}$ linearly on each simplex of the triangulation $\mathcal{K}$ to the embedding $i_{P}: P^{n} \hookrightarrow I^{m}$.

Figure 1 describes the embedding $i_{P}: P^{n} \hookrightarrow I^{m}$ in the case $n=2, m=3$.

The embedding $i_{P}: P^{n} \hookrightarrow I^{m}$ has the following property:

Proposition 1.5. If $v=F_{i_{1}}^{n-1} \cap \cdots \cap F_{i_{n}}^{n-1}$ is a vertex of $P^{n}$, then the cube $I_{v}^{n} \subset P^{n}$ is mapped onto the $n$-face of the cube $I^{m}$ determined by $m-n$ equations $y_{j}=1, j \notin\left\{i_{1}, \ldots, i_{n}\right\}$.

Now, let us consider the standard unit poly-disk

$$
\left(D^{2}\right)^{m}=\left\{\left(z_{1}, \ldots, z_{m}\right) \in \mathbb{C}^{m}:\left|z_{i}\right| \leq 1\right\} \subset \mathbb{C}^{m} .
$$

The standard action of $T^{m}$ on $\mathbb{C}^{m}$ by diagonal matrices defines the action of $T^{m}$ on $\left(D^{2}\right)^{m}$ with orbit space $I^{m}$.

THEOREM 1.6. The space $\mathcal{Z}_{P}$ has a canonical structure of smooth $(m+n)$ dimensional manifold such that the $T^{m}$-action is smooth. The embedding $i_{P}: P^{n} \hookrightarrow$ $I^{m}$ constructed in Lemma 1.4 is covered by a $T^{m}$-equivariant embedding $i_{e}: \mathcal{Z}_{P} \hookrightarrow$ 
$\left(D^{2}\right)^{m} \subset \mathbb{C}^{m}$. This can be described by the commutative diagram

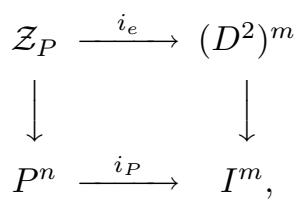

Proof. Let $\rho: \mathcal{Z}_{P} \rightarrow P^{n}$ be the orbit map. It easily follows from the definition of $\mathcal{Z}_{P}$ that for each cube $I_{v}^{n} \subset P^{n}$ (see Lemma 1.4) we have $\rho^{-1}\left(I_{v}^{n}\right) \cong\left(D^{2}\right)^{n} \times$ $T^{m-n}$. Here $\left(D^{2}\right)^{n}$ is the unit poly-disk in $\mathbb{C}^{n}$ with diagonal action of $T^{n}$. Hence, $\mathcal{Z}_{P}$ is represented as the union of blocks $B_{v}=\rho^{-1}\left(I_{v}^{n}\right)$, each of which is isomorphic to $\left(D^{2}\right)^{n} \times T^{m-n}$. These blocks $B_{v}$ are glued together along their boundaries to get the smooth $T^{m}$-manifold $\mathcal{Z}_{P}$.

Now, let us prove the second part of the theorem concerning the equivariant embedding. First, we fix a numeration of codimension-one faces of $P^{n}$ : $F_{1}^{n-1}, \ldots, F_{m}^{n-1}$. Take the block

$$
B_{v} \cong\left(D^{2}\right)^{n} \times T^{m-n}=D^{2} \times \ldots \times D^{2} \times S^{1} \times \ldots \times S^{1}
$$

corresponding to a vertex $v \in P^{n}$. Each factor $D^{2}$ or $T^{1}$ in $B_{v}$ corresponds to a codimension-one face of $P^{n}$ and therefore acquires a number (index) $i, 1 \leq i \leq m$. Note that $n$ factors $D^{2}$ acquire the indices corresponding to those codimension-one faces containing $v$, while other indices are assigned to $m-n$ factors $T^{1}$. Now we numerate the factors $D^{2} \subset\left(D^{2}\right)^{m}$ of the poly-disk in any way and embed each block $B_{v} \subset \mathcal{Z}_{P}$ into $\left(D^{2}\right)^{m}$ according to the indexes of its factors. It can be easily seen that the embedding of a face $I^{n}$ given by $m-n$ equations of type $y_{j}=1$ (as in Proposition 1.5) into the cube $I^{m}$ is covered by the above constructed embedding of $B_{v} \cong\left(D^{2}\right)^{n} \times T^{m-n}$ into $\left(D^{2}\right)^{m}$. Then it follows from Proposition 1.5 that the set of embeddings $\left(D^{2}\right)^{n} \times T^{m-n} \cong B_{v} \hookrightarrow\left(D^{2}\right)^{m}$ defines an equivariant embedding $i_{e}: \mathcal{Z}_{P} \hookrightarrow\left(D^{2}\right)^{m}$. By the construction, this embedding covers the embedding $i_{P}: P^{n} \hookrightarrow I^{m}$ from Lemma 1.4.

EXAMPLE 1.7. If $P^{n}=\Delta^{1}$ is an 1-dimensional simplex (a segment), then $B_{v}=$ $D^{2} \times S^{1}$ for each of the two vertices, and we obtain the well-known decomposition $\mathcal{Z}_{\Delta^{1}} \cong S^{3}=D^{2} \times S^{1} \cup S^{1} \times D^{2}$. If $P^{n}=\Delta^{n}$ is an $n$-dimensional simplex, we obtain the similar decomposition of a $(2 n+1)$-sphere into $n+1$ "blocks" $\left(D^{2}\right)^{n} \times S^{1}$.

\section{Connections with toric varieties and subspace arrangements}

The above constructed embedding $i_{e}: \mathcal{Z}_{P} \hookrightarrow\left(D^{2}\right)^{m} \subset \mathbb{C}^{m}$ allows us to connect the manifold $\mathcal{Z}_{P}$ with one construction from the theory of toric varieties. Below we describe this construction, following $[\mathbf{B a}]$.

Definition 2.1. Let $I=\left\{i_{1}, \ldots, i_{p}\right\}$ be an index set, and let $A_{I} \subset \mathbb{C}^{m}$ denote the coordinate subspace $z_{i_{1}}=\cdots=z_{i_{p}}=0$. Define the arrangement $\mathbf{A}\left(P^{n}\right)$ of subspaces of $\mathbb{C}^{m}$ as

$$
\mathbf{A}\left(P^{n}\right)=\bigcup_{I} A_{I},
$$

where the union is taken over all $I=\left\{i_{1}, \ldots, i_{p}\right\}$ such that $F_{i_{1}} \cap \cdots \cap F_{i_{p}}=\varnothing$ in $P^{n}$. Put

$$
U\left(P^{n}\right)=\mathbb{C}^{m} \backslash \mathbf{A}\left(P^{n}\right) .
$$


Note that the closed set $\mathbf{A}\left(P^{n}\right)$ has codimension at least 2 and is invariant with respect to the diagonal action of $\left(\mathbb{C}^{*}\right)^{m}$ on $\mathbb{C}^{m}$. (Here $\mathbb{C}^{*}$ denote the multiplicative group of non-zero complex numbers). Hence, $\left(\mathbb{C}^{*}\right)^{m}$, as well as the torus $T^{m} \subset$ $\left(\mathbb{C}^{*}\right)^{m}$, acts on $U\left(P^{n}\right) \subset \mathbb{C}^{m}$.

It follows from Proposition 1.5 that the image of $\mathcal{Z}_{P}$ under the embedding $i_{e}$ : $\mathcal{Z}_{P} \rightarrow \mathbb{C}^{m}$ (see Theorem 1.6) does not intersect $\mathbf{A}\left(P^{n}\right)$, that is, $i_{e}\left(\mathcal{Z}_{P}\right) \subset U\left(P^{n}\right)$.

We put

$$
\mathbb{R}_{>}^{m}=\left\{\left(\alpha_{1}, \ldots, \alpha_{m}\right) \in \mathbb{R}^{n}: \alpha_{i}>0\right\}
$$

This is a group with respect to multiplication, which acts by dilations on $\mathbb{R}^{m}$ and $\mathbb{C}^{m}$ (an element $\left(\alpha_{1}, \ldots, \alpha_{m}\right) \in \mathbb{R}_{>}^{m}$ takes $\left(y_{1}, \ldots, y_{m}\right) \in \mathbb{R}^{m}$ to $\left.\left(\alpha_{1} y_{1}, \ldots, \alpha_{m} y_{m}\right)\right)$. There is the isomorphism exp : $\mathbb{R}^{m} \rightarrow \mathbb{R}_{>}^{m}$ between additive and multiplicative groups, which takes $\left(t_{1}, \ldots, t_{m}\right) \in \mathbb{R}^{m}$ to $\left(e^{t_{1}}, \ldots, e^{t_{m}}\right) \in \mathbb{R}_{>}^{m}$.

Remember that the polytope $P^{n}$ is a set of points $x \in \mathbb{R}^{n}$ satisfying $m$ linear inequalities:

$$
P^{n}=\left\{x \in \mathbb{R}^{n}:\left\langle l_{i}, x\right\rangle \geq-a_{i}, i=1, \ldots, m\right\},
$$

where $l_{i} \in\left(\mathbb{R}^{n}\right)^{*}$ are normal (co)vectors of facets. The set of $\left(\mu_{1}, \ldots, \mu_{m}\right) \in \mathbb{R}^{m}$ such that $\mu_{1} l_{1}+\ldots+\mu_{m} l_{m}=0$ is an $(m-n)$-dimensional subspace in $\mathbb{R}^{m}$. We choose a basis $\left\{w_{i}=\left(w_{1 i}, \ldots, w_{m i}\right)^{\top}\right\}, 1 \leq i \leq m-n$, in this subspace and form the $m \times(m-n)$-matrix

$$
W=\left(\begin{array}{ccc}
w_{11} & \ldots & w_{1, m-n} \\
\ldots & \ldots & \ldots \\
w_{m 1} & \ldots & w_{m, m-n}
\end{array}\right)
$$

of maximal rank $m-n$. This matrix satisfies the following property.

Proposition 2.2. Suppose that $n$ facets $F_{i_{1}}^{n-1}, \ldots, F_{i_{n}}^{n-1}$ of $P^{n}$ meet at the same vertex $v: F_{i_{1}}^{n-1} \cap \cdots \cap F_{i_{n}}^{n-1}=v$. Then the minor $(m-n) \times(m-n)$ matrix $W_{i_{1} \ldots i_{n}}$ obtained from $W$ by deleting $n$ rows $i_{1}, \ldots, i_{n}$ is non-degenerate: $\operatorname{det} W_{i_{1} \ldots i_{n}} \neq 0$.

Proof. Suppose det $W_{i_{1}, \ldots, i_{n}}=0$, then one can find a zero non-trivial linear combination of vectors $l_{i_{1}}, \ldots, l_{i_{n}}$. But this is impossible: since $P^{n}$ is simple, the set of normal vectors of facets meeting at the same vertex constitute a basis of $\mathbb{R}^{n}$.

The matrix $W$ defines the subgroup

$$
R_{W}=\left\{\left(e^{w_{11} \tau_{1}+\cdots+w_{1, m-n} \tau_{m-n}}, \ldots, e^{w_{m 1} \tau_{1}+\cdots+w_{m, m-n} \tau_{m-n}}\right) \in \mathbb{R}_{>}^{m}\right\} \subset \mathbb{R}_{>}^{m},
$$

where $\left(\tau_{1}, \ldots, \tau_{m-n}\right)$ runs over $\mathbb{R}^{m-n}$. This subgroup is isomorphic to $\mathbb{R}_{>}^{m-n}$. Since $U\left(P^{n}\right) \subset \mathbb{C}^{m}$ (see Definition 2.1) is invariant with respect to the action of $\mathbb{R}_{>}^{m} \subset$ $\left(\mathbb{C}^{*}\right)^{m}$ on $\mathbb{C}^{m}$, the subgroup $R_{W} \subset \mathbb{R}_{>}^{m}$ also acts on $U\left(P^{n}\right)$.

TheOREM 2.3. The subgroup $R_{W} \subset \mathbb{R}_{>}^{m}$ acts freely on $U\left(P^{n}\right) \subset \mathbb{C}^{m}$. The composite map $\mathcal{Z}_{P} \rightarrow U\left(P^{n}\right) \rightarrow U\left(P^{n}\right) / R_{W}$ of the embedding $i_{e}$ and the orbit map is a homeomorphism.

Proof. A point from $\mathbb{C}^{m}$ may have the non-trivial isotropy subgroup with respect to the action of $\mathbb{R}_{>}^{m}$ on $\mathbb{C}^{m}$ only if at least one of its coordinates vanishes. As it follows from Definition 2.1, if a point $x \in U\left(P^{n}\right)$ has some zero coordinates, then all of them correspond to facets of $P^{n}$ having at least one common vertex $v \in P^{n}$. Let $v=F_{i_{1}}^{n-1} \cap \cdots \cap F_{i_{n}}^{n-1}$. Then the isotropy subgroup of the point $x$ 
with respect to the action of $R_{W}$ is non-trivial only if some linear combination of vectors $w_{1}, \ldots, w_{m-n}$ lies in the coordinate subspace spanned by $e_{i_{1}}, \ldots, e_{i_{n}}$. But this means that $\operatorname{det} W_{i_{1} \ldots i_{n}}=0$, which contradicts Proposition 2.2. Thus, $R_{W}$ acts freely on $U\left(P^{n}\right)$.

Now, let us prove the second part of the theorem. Here we use both embeddings $i_{e}: \mathcal{Z}_{P} \rightarrow\left(D^{2}\right)^{m} \subset \mathbb{C}^{m}$ from Theorem 1.6 and $i_{P}: P^{n} \rightarrow I^{m} \subset \mathbb{R}^{m}$ from Lemma 1.4. It is sufficient to prove that each orbit of the action of $R_{W}$ on $U\left(P^{n}\right) \subset \mathbb{C}^{m}$ intersects the image $i_{e}\left(\mathcal{Z}_{P}\right)$ at a single point. Since the embedding $i_{e}$ is equivariant, instead of this we may prove that each orbit of the action of $R_{W}$ on the real part $U_{\mathbb{R}}\left(P^{n}\right)=U\left(P^{n}\right) \cap \mathbb{R}_{+}^{m}$ intersects the image $i_{P}\left(P^{n}\right)$ in a single point. Let $y \in i_{P}\left(P^{n}\right) \subset \mathbb{R}^{m}$. Then $y=\left(y_{1}, \ldots, y_{m}\right)$ lies in some $n$-face $I_{v}^{n}$ of the unit cube $I^{m} \subset \mathbb{R}^{m}$ as described by Proposition 1.5. We need to show that the $(m-n)$-dimensional subspace spanned by the vectors $\left(w_{11} y_{1}, \ldots, w_{m 1} y_{m}\right)^{\top}, \ldots,\left(w_{1, m-n} y_{1}, \ldots, w_{m, m-n} y_{m}\right)^{\top}$ is in general position with the $n$-face $I_{v}^{n}$. But this follows directly from Propositions 1.5 and 2.2.

The above theorem gives a new proof of the fact that $\mathcal{Z}_{P}$ is a smooth manifold, which allows a $T^{m}$-equivariant embedding into $\mathbb{C}^{m} \cong \mathbb{R}^{2 m}$ with trivial normal bundle.

EXAMPLE 2.4. Let $P^{n}=\Delta^{n}$ (an $n$-simplex). Then $m=n+1, U\left(P^{n}\right)=$ $\mathbb{C}^{n+1} \backslash\{0\}, R_{>}^{m-n}$ is $\mathbb{R}_{>}$, and $\alpha \in \mathbb{R}_{>}$takes $z \in \mathbb{C}^{n+1}$ to $\alpha z$. Thus, we have $\mathcal{Z}_{P}=S^{2 n+1}$ (this could be also deduced from Definition 1.2; see also Example 1.7).

Now, suppose that all vertices of $P^{n}$ belong to the integer lattice $\mathbb{Z}^{n} \subset \mathbb{R}^{n}$. Such an integral simple polytope $P^{n}$ defines a projective toric variety $M_{P}$ (see $\left.[\mathbf{F u}]\right)$. Normal (co)vectors $l_{i}$ of facets of $P^{n}$ (see (2.1)) can be taken integral and primitive. The toric variety $M_{P}$ defined by $P^{n}$ is smooth if for each vertex $v=F_{i_{1}} \cap \ldots \cap F_{i_{n}}$ the vectors $l_{i_{1}}, \ldots, l_{i_{n}}$ constitute an integral basis of $\mathbb{Z}^{n}$. As before, we may construct the matrix $W$ (see (2.2)) and then define the subgroup

$$
C_{W}=\left\{\left(e^{w_{11} \tau_{1}+\cdots+w_{1, m-n} \tau_{m-n}}, \ldots, e^{w_{m 1} \tau_{1}+\cdots+w_{m, m-n} \tau_{m-n}}\right)\right\} \subset\left(\mathbb{C}^{*}\right)^{m},
$$

where $\left(\tau_{1}, \ldots, \tau_{m-n}\right)$ runs over $\mathbb{C}^{m-n}$. This subgroup is isomorphic to $\left(\mathbb{C}^{*}\right)^{m-n}$. It can be shown (see $[\mathbf{B a}]$ ) that $C_{W}$ acts freely on $U\left(P^{n}\right)$ and the toric manifold $M_{P}$ is identified with the orbit space (or the geometric quotient) $U\left(P^{n}\right) / C_{W}$. Thus, we have the commutative diagram

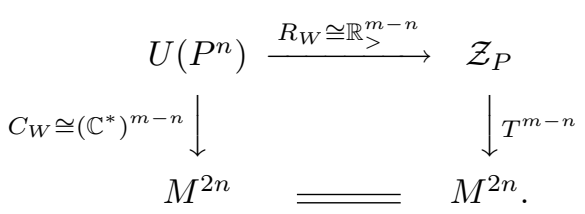

Since $\mathcal{Z}_{P}$ can be viewed as the orbit space of $U\left(P^{n}\right)$ with respect to the action of $R_{W} \cong \mathbb{R}_{>}^{m-n}$, the manifold $\mathcal{Z}_{P}$ and the complement $U\left(P^{n}\right)$ of an arrangement of planes are of same homotopy type. In the next section we calculate the cohomology ring of $\mathcal{Z}_{P}\left(\right.$ or $\left.U\left(P^{n}\right)\right)$.

\section{Cohomology ring of $\mathcal{Z}_{P}$}

The following lemma follows readily from the construction of $\mathcal{Z}_{P}$. 
LEMMA 3.1. If $P^{n}$ is the product of two simple polytopes: $P^{n}=P_{1}^{n_{1}} \times P_{2}^{n_{2}}$, then $\mathcal{Z}_{P}=\mathcal{Z}_{P_{1}} \times \mathcal{Z}_{P_{2}}$. If $P_{1}^{n_{1}} \subset P^{n}$ is a face, then $\mathcal{Z}_{P_{1}}$ is a submanifold of $\mathcal{Z}_{P}$.

The space $B T^{m}=\left(\mathbb{C} P^{\infty}\right)^{m}$ has a canonical cellular decomposition (that is, each $\mathbb{C} P^{\infty}$ has one cell in each even dimension). For each index set $I=\left\{i_{1}, \ldots, i_{k}\right\}$ we introduce the cellular subcomplex $B T_{I}^{k}=B T_{i_{1}, \ldots, i_{k}}^{k} \subset B T^{m}$ homeomorphic to $B T^{k}$.

Definition 3.2. Define the cellular subcomplex $\widetilde{B_{T} P} \subset B T^{m}$ to be the union of $B T_{I}^{k}$ over all $I=\left\{i_{1}, \ldots, i_{k}\right\}$ such that $F_{i_{1}} \cap \cdots \cap F_{i_{p}} \neq \varnothing$ in $P^{n}$.

THEOREM 3.3. The cellular embedding $i: \widetilde{B_{T} P} \hookrightarrow B T^{m}$ (see Definition 3.2) and the fibration $p: B_{T} P \rightarrow B T^{m}$ (see (1.3)) are homotopically equivalent. In particular, $\widetilde{B_{T} P}$ and $B_{T} P$ are of same homotopy type.

Proof. The proof can be found in [BP1].

COROLlary 3.4. The cohomology ring of $B_{T} P$ is isomorphic to the face ring $\mathbf{k}\left(P^{n}\right)$ (see Definition 1.1). The projection $p: B_{T} P \hookrightarrow B T^{m}$ induces the quotient epimorphism $p^{*}: \mathbf{k}\left[v_{1}, \ldots, v_{m}\right] \rightarrow \mathbf{k}\left(P^{n}\right)=\mathbf{k}\left[v_{1}, \ldots, v_{m}\right] / I$ in the cohomology.

A simple polytope $P^{n}$ with $m$ codimension-one faces is called $q$-neighbourly $[\mathbf{B r}]$ if the $(q-1)$-skeleton of the simplicial complex $K_{P}^{n-1}$ dual to the boundary $\partial P^{n}$ coincides with the $(q-1)$-skeleton of an $(m-1)$-simplex. Equivalently, $P^{n}$ is $q$ neighbourly if any $q$ codimension-one faces of $P^{n}$ have non-empty intersection. Note that any simple polytope is 1-neighbourly. The next theorem about the homotopy groups of $\mathcal{Z}_{P}$ and $B_{T} P$ follows easily from cellular structure of $B_{T} P$ and exact homotopy sequence of the bundle $p: B_{T} P \rightarrow B T^{m}$ with fibre $\mathcal{Z}_{P}$.

THEOREM 3.5. For any simple polytope $P^{n}$ with $m$ codimension-one faces we have:

(1) $\pi_{1}\left(\mathcal{Z}_{P}\right)=\pi_{1}\left(B_{T} P\right)=0$.

(2) $\pi_{2}\left(\mathcal{Z}_{P}\right)=0, \pi_{2}\left(B_{T} P\right)=\mathbb{Z}^{m}$.

(3) $\pi_{q}\left(\mathcal{Z}_{P}\right)=\pi_{q}\left(B_{T} P\right)$ for $q \geq 3$.

(4) If $P^{n}$ is q-neighbourly, then $\pi_{i}\left(\mathcal{Z}_{P}\right)=0$ for $i<2 q+1$, and $\pi_{2 q+1}\left(\mathcal{Z}_{P}\right)$ is a free Abelian group whose generators correspond to monomials $v_{i_{1}} \cdots v_{i_{q+1}} \in I$ (see Definition 1.1).

From (1.3) we obtain the commutative square

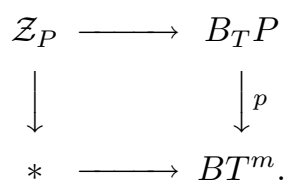

The Eilenberg-Moore spectral sequence $[\mathbf{S m}]$ of this square has the $E_{2}$-term

$$
E_{2} \cong \operatorname{Tor}_{\mathbf{k}\left[v_{1}, \ldots, v_{m}\right]}\left(\mathbf{k}\left(P^{n}\right), \mathbf{k}\right)
$$

where $\mathbf{k}\left(P^{n}\right)$ is regarded as a $\mathbf{k}\left[v_{1}, \ldots, v_{m}\right]$-module by means of quotient projection $\mathbf{k}\left[v_{1}, \ldots, v_{m}\right] \rightarrow \mathbf{k}\left[v_{1}, \ldots, v_{m}\right] / I=\mathbf{k}\left(P^{n}\right)$. This spectral sequence converges to the cohomology of $\mathcal{Z}_{P}$. It turns out that the spectral sequence collapses at the $E_{2}$ term, and moreover, the following statement holds: 
Theorem 3.6. Provided that $\mathbf{k}$ is a field, we have an isomorphism of algebras:

$$
H^{*}\left(\mathcal{Z}_{P}\right) \cong \operatorname{Tor}_{\mathbf{k}\left[v_{1}, \ldots, v_{m}\right]}\left(\mathbf{k}\left(P^{n}\right), \mathbf{k}\right) .
$$

The additive structure of the cohomology is thus given by the isomorphisms

$$
H^{r}\left(\mathcal{Z}_{P}\right) \cong \bigoplus_{2 j-i=r} \operatorname{Tor}_{\mathbf{k}\left[v_{1}, \ldots, v_{m}\right]}^{-i, 2 j}\left(\mathbf{k}\left(P^{n}\right), \mathbf{k}\right), \quad i, j \geq 0 .
$$

Proof. The proof of the theorem uses some results of $[\mathbf{S m}]$ on the EilenbergMoore spectral sequences and Theorem 3.3. This proof can be found in [BP1].

Suppose that there is at least one smooth toric variety $M^{2 n}$ whose orbit space with respect to the $T^{n}$-action has combinatorial type of the simple polytope $P^{n}$. Then one can find a subgroup $T^{m-n} \cong H \subset T^{m}$ that acts freely on the manifold $\mathcal{Z}_{P}$ such that $M^{2 n}=\mathcal{Z}_{P} / H$. In general case such a subgroup may fail to exist; however, sometimes one can find a subgroup of dimension less than $m-n$ that acts freely on $\mathcal{Z}_{P}$. So, let $H \cong T^{r}$ be such a subgroup. Then the inclusion $s: H \hookrightarrow T^{m}$ is determined by an integral $(m \times r)$-matrix $\left(s_{i j}\right)$ such that the $\mathbb{Z}$-module spanned by its columns $s_{j}=\left(s_{1 j}, \ldots, s_{m j}\right)^{\top}, j=1, \ldots, r$, is a direct summand in $\mathbb{Z}^{m}$. Choose any basis $t_{i}=\left(t_{i 1}, \ldots, t_{i m}\right), i=1, \ldots, m-r$, in the kernel of the dual map $s^{*}:\left(\mathbb{Z}^{m}\right)^{*} \rightarrow\left(\mathbb{Z}^{r}\right)^{*}$. Then we have the following result describing the cohomology ring of the manifold $\mathcal{Y}=\mathcal{Z}_{P} / H$.

THEOREM 3.7. The following isomorphism of algebras holds:

$$
H^{*}(\mathcal{Y}) \cong \operatorname{Tor}_{\mathbf{k}\left[t_{1}, \ldots, t_{m-r}\right]}\left(\mathbf{k}\left(P^{n}\right), \mathbf{k}\right),
$$

where the $\mathbf{k}\left[t_{1}, \ldots, t_{m-r}\right]$-module structure on $\mathbf{k}\left(P^{n}\right)=\mathbf{k}\left[v_{1}, \ldots, v_{m}\right] / I$ is defined by the map

$$
\begin{aligned}
\mathbf{k}\left[t_{1}, \ldots, t_{m-r}\right] & \rightarrow \mathbf{k}\left[v_{1}, \ldots, v_{m}\right] \\
t_{i} & \rightarrow t_{i 1} v_{1}+\ldots+t_{i m} v_{m} .
\end{aligned}
$$

Proof. This theorem, as well as the previous one, can be proved by considering a certain Eilenberg-Moore spectral sequence. See [BP1, Theorem 4.13].

In the case of toric varieties (that is, $r=m-n$ in Theorem 3.7) we obtain

$$
H^{*}\left(M^{2 n}\right) \cong \operatorname{Tor}_{\mathbf{k}\left[t_{1}, \ldots, t_{n}\right]}\left(\mathbf{k}\left(P^{n}\right), \mathbf{k}\right) .
$$

It can be shown that in this case $\mathbf{k}\left(P^{n}\right)$ is a free $\mathbf{k}\left[t_{1}, \ldots, t_{n}\right]$-module (which implies that $t_{1}, \ldots, t_{n}$ is a regular sequence, and $\mathbf{k}\left(P^{n}\right)$ is a so-called Cohen-Macaulay ring). Thus, we have

$$
H^{*}\left(M^{2 n}\right) \cong \mathbf{k}\left(P^{n}\right) / J=\mathbf{k}\left[v_{1}, \ldots, v_{m}\right] / I+J,
$$

where $J$ is the ideal generated by $t_{i 1} v_{1}+\ldots+t_{i m} v_{m}, i=1, \ldots, n$. This result (the description of the cohomology ring of a smooth toric variety) is well known in algebraic geometry as the Danilov-Jurkiewicz theorem [Da].

In order to describe the cohomology ring of $\mathcal{Z}_{P}$ more explicitly, we apply some constructions from homological algebra.

Let $\Gamma=\mathbf{k}\left[y_{1}, \ldots, y_{n}\right], \operatorname{deg} y_{i}=2$, be a graded polynomial algebra, and let $A$ be any graded $\Gamma$-module. Let $\Lambda\left[u_{1}, \ldots, u_{n}\right]$ denote the exterior algebra on generators $u_{1}, \ldots, u_{n}$ over $\mathbf{k}$, and consider the complex

$$
\mathcal{E}=\Gamma \otimes \Lambda\left[u_{1}, \ldots, u_{n}\right] .
$$


This is a bigraded differential algebra; its gradings and differential are defined by

$$
\begin{aligned}
\operatorname{bideg}\left(y_{i} \otimes 1\right) & =(0,2), & & d\left(y_{i} \otimes 1\right)=0 ; \\
\operatorname{bideg}\left(1 \otimes u_{i}\right) & =(-1,2), & & d\left(1 \otimes u_{i}\right)=y_{i} \otimes 1
\end{aligned}
$$

and requiring that $d$ be a derivation of algebras. The differential adds $(1,0)$ to bidegree, hence, the components $\mathcal{E}^{-i, *}$ form a cochain complex. It is well known that this complex is a $\Gamma$-free resolution of $\mathbf{k}$ (regarded as a $\Gamma$-module) called the Koszul resolution (see $[\mathbf{M a}]$ ). Thus, for any $\Gamma$-module $A$ we have

$$
\operatorname{Tor}_{\Gamma}(A, \mathbf{k})=H\left[A \otimes_{\Gamma} \Gamma \otimes \Lambda\left[u_{1}, \ldots, u_{n}\right], d\right]=H\left[A \otimes \Lambda\left[u_{1}, \ldots, u_{n}\right], d\right],
$$

where $d$ is defined as $d\left(1 \otimes u_{i}\right)=y_{i} \otimes 1$.

Applying this construction to the case $\Gamma=\mathbf{k}\left[v_{1}, \ldots, v_{m}\right], A=\mathbf{k}\left(P^{n}\right)$ and using Theorem 3.6, we get the following statement.

THEOREM 3.8. The following isomorphism of graded algebras holds:

$$
\begin{gathered}
H^{*}\left(\mathcal{Z}_{P}\right) \cong H\left[\mathbf{k}\left(P^{n}\right) \otimes \Lambda\left[u_{1}, \ldots, u_{m}\right], d\right], \\
\operatorname{bideg} v_{i}=(0,2), \quad \operatorname{bideg} u_{i}=(-1,2), \\
d\left(1 \otimes u_{i}\right)=v_{i} \otimes 1, \quad d\left(v_{i} \otimes 1\right)=0,
\end{gathered}
$$

where $\mathbf{k}\left(P^{n}\right)=\mathbf{k}\left[v_{1}, \ldots, v_{m}\right] / I$ is the face ring.

Corollary 3.9. The Leray-Serre spectral sequence of the $T^{m}$-bundle

$$
\mathcal{Z}_{P} \times E T^{m} \rightarrow B_{T} P=\mathcal{Z}_{P} \times_{T^{m}} E T^{m}
$$

collapses at the $E_{3}$ term.

Theorems 3.6 and 3.8 show that instead of usual grading, the cohomology of $\mathcal{Z}_{P}$ has bigraded algebra structure with bigraded components

$$
H^{-i, 2 j}\left(\mathcal{Z}_{P}\right) \cong \operatorname{Tor}_{\mathbf{k}\left[v_{1}, \ldots, v_{m}\right]}^{-i, 2 j}\left(\mathbf{k}\left(P^{n}\right), \mathbf{k}\right), \quad i, j \geq 0,
$$

satisfying $H^{r}\left(\mathcal{Z}_{P}\right)=\bigoplus_{2 j-i=r} H^{-i, 2 j}\left(\mathcal{Z}_{P}\right)$.

Since $\mathcal{Z}_{P}$ is a manifold, there is the Poincaré duality in $H^{*}\left(\mathcal{Z}_{P}\right)$. This Poincaré duality has the following combinatorial interpretation.

LEMMA 3.10 .

(1) The Poincaré duality in $H^{*}\left(\mathcal{Z}_{P}\right)$ regards the bigraded structure defined by theorems 3.6 and 3.8. More precisely, if $\alpha \in H^{-i, 2 j}\left(\mathcal{Z}_{P}\right)$ is a cohomology class, then its Poincaré dual D $\alpha$ belongs to $H^{-(m-n)+i, 2(m-j)}$.

(2) Let $v=F_{i_{1}}^{n-1} \cap \cdots \cap F_{i_{n}}^{n-1}$ be a vertex of the polytope $P^{n}$, and let $j_{1}<$ $\ldots<j_{m-n},\left\{i_{1}, \ldots, i_{n}, j_{1}, \ldots, j_{m-n}\right\}=\{1, \ldots, m\}$. Then the value of the element

$$
v_{i_{1}} \cdots v_{i_{n}} u_{j_{1}} \cdots u_{j_{m-n}} \in H^{m+n}\left(\mathcal{Z}_{P}\right)
$$

on the fundamental class of $\mathcal{Z}_{P}$ equals \pm 1 .

(3) Let $v_{1}=F_{i_{1}}^{n-1} \cap \cdots \cap F_{i_{n}}^{n-1}$ and $v_{2}=F_{i_{1}}^{n-1} \cap \cdots \cap F_{i_{n-1}}^{n-1} \cap F_{j}^{n-1}$ be two vertices of $P^{n}$ connected by an edge, and $j_{1}, \ldots, j_{m-n}$ as above. Then

$$
v_{i_{1}} \cdots v_{i_{n}} u_{j_{1}} \cdots u_{j_{m-n}}=v_{i_{1}} \cdots v_{i_{n-1}} v_{j_{1}} u_{i_{n}} u_{j_{2}} \cdots u_{j_{m-n}}
$$

in $H^{m+n}\left(\mathcal{Z}_{P}\right)$. 
Proof. The second assertion follows from the fact that the cohomology class under consideration is a generator of the module $\operatorname{Tor}_{\mathbf{k}\left[v_{1}, \ldots, v_{m}\right]}^{-(m-n), m}\left(\mathbf{k}\left(P^{n}\right), \mathbf{k}\right)=$ $H^{m+n}\left(\mathcal{Z}_{P}^{m+n}\right)$ (see Theorem 3.6). To prove the third assertion we just mention that

$$
\begin{aligned}
d\left(v_{i_{1}} \cdots v_{i_{n-1}} u_{i_{n}} u_{j_{1}} u_{j_{2}} \cdots u_{j_{m-n}}\right) & \\
& =v_{i_{1}} \cdots v_{i_{n}} u_{j_{1}} \cdots u_{j_{m-n}}-v_{i_{1}} \cdots v_{i_{n-1}} v_{j_{1}} u_{i_{n}} u_{j_{2}} \cdots u_{j_{m-n}}
\end{aligned}
$$

in $\mathbf{k}\left(P^{n}\right) \otimes \Lambda\left[u_{1}, \ldots, u_{m}\right]$ (see Theorem 3.8).

Changing the numeration of codimension-one faces of $P^{n}$ and the orientation of $\mathcal{Z}_{P}$ if necessary, we may assume that the fundamental cohomology class of $\mathcal{Z}_{P}$ is represented by the cocycle $v_{1} \cdots v_{n} u_{m+1} \cdots u_{m} \in \mathbf{k}\left(P^{n}\right) \otimes \Lambda\left[u_{1}, \ldots, u_{m}\right]$.

\section{New relations between combinatorics and topology}

The results on the topology of manifolds defined by simple polytopes obtained in the previous sections give rise to new remarkable connections with combinatorics of polytopes. Here we discuss only few examples.

Set $\mathcal{T}^{i}=\operatorname{Tor}_{\mathbf{k}\left[v_{1}, \ldots, v_{m}\right]}^{-i}\left(\mathbf{k}\left(P^{n}\right), \mathbf{k}\right)$ and $\mathcal{T}^{i, j}=\operatorname{Tor}_{\mathbf{k}\left[v_{1}, \ldots, v_{m}\right]}^{-i, j}\left(\mathbf{k}\left(P^{n}\right), \mathbf{k}\right)$. Then Lemma 3.10 shows that the Poincaré duality for $\mathcal{Z}_{P}$ can be shortly written as the following identity for the Poincaré series $F\left(\mathcal{T}^{i}, t\right)=\sum_{r=0}^{m} \operatorname{dim}_{\mathbf{k}}\left(\mathcal{T}^{i, 2 r}\right) t^{2 r}$ of $\mathcal{T}^{i}$ :

$$
F\left(\mathcal{T}^{i}, t\right)=t^{2 m} F\left(\mathcal{T}^{m-n-i}, \frac{1}{t}\right) .
$$

The above identity is well known in commutative algebra for so-called Gorenstein simplicial complexes (see [St2, p. 76]). A simplicial complex $K$ with $m$ vertices is Gorenstein over $\mathbf{k}$ if the face ring $\mathbf{k}(K)$ is Cohen-Macaulay and

$$
\operatorname{dim} \operatorname{Tor}_{\mathbf{k}\left[v_{1}, \ldots, v_{m}\right]}^{-(m-n)}(\mathbf{k}(K), \mathbf{k})=1,
$$

where $n$ is the maximal number of algebraically independent elements of $\mathbf{k}(K)$, that is, the maximal number of vertices of simplices of $K$. It is known that the face ring of simplicial subdivision of a sphere $S^{n-1}$ is Gorenstein (see [St2, p. 76]). In particular, our face ring $\mathbf{k}\left(P^{n}\right)$ of simple polytope $P^{n}$ is Gorenstein, and the maximal number of algebraically independent elements of $\mathbf{k}\left(P^{n}\right)$ equals the dimension of $P^{n}$.

A simple combinatorial argument (see [St2, part II, §1]) shows that for any simple polytope $P^{n}$ the Poincaré series $F\left(\mathbf{k}\left(P^{n}\right), t\right)$ can be written as follows

$$
F\left(\mathbf{k}\left(P^{n}\right), t\right)=1+\sum_{i=0}^{n-1} \frac{f_{i} t^{2(i+1)}}{\left(1-t^{2}\right)^{i+1}},
$$

where $\left(f_{0}, \ldots, f_{n-1}\right)$ is the $f$-vector of $P$. This series can be also expressed in terms of the $h$-vector $\left(h_{0}, \ldots, h_{n}\right)$ (see $\left.(1.1)\right)$ as

$$
F\left(\mathbf{k}\left(P^{n}\right), t\right)=\frac{h_{0}+h_{1} t^{2}+\ldots+h_{n} t^{2 n}}{\left(1-t^{2}\right)^{n}} .
$$

On the other hand, one can also deduce the formula for $F\left(\mathbf{k}\left(P^{n}\right), t\right)$ from the Hilbert syzygy theorem by applying it to the minimal resolution of $\mathbf{k}\left(P^{n}\right)$ regarded as a $\mathbf{k}\left[v_{1}, \ldots, v_{m}\right]$-module. This formula is as follows

$$
F\left(\mathbf{k}\left(P^{n}\right), t\right)=\frac{\sum_{i=0}^{m}(-1)^{i} F\left(\mathcal{T}^{i}, t\right)}{\left(1-t^{2}\right)^{m}} .
$$


Combining (4.1), (4.2), and (4.3) we get

$$
h_{i}=h_{n-i}, \quad i=0,1, \ldots, n .
$$

These are the well-known Dehn-Sommerville equations (see, for example, $[\mathbf{B r}]$ ) for simple (or simplicial) polytopes. Dehn-Sommerville equations also hold for any Gorenstein simplicial complex $K$ (that is, such that the face ring $\mathbf{k}(K)$ is Gorenstein, see [St2, p. 77]). Thus, we see that the algebraic duality (4.1) for Gorenstein simplicial complexes and the combinatorial Dehn-Sommerville equations (4.4) follow from the Poincaré duality for the manifold $\mathcal{Z}_{P}$.

Now, we define the bigraded Betti numbers $b^{-i, 2 j}$ as

$$
b^{-i, 2 j}=\operatorname{dim}_{\mathbf{k}} \operatorname{Tor}_{\mathbf{k}\left[v_{1}, \ldots, v_{m}\right]}^{-i, 2 j}\left(\mathbf{k}\left(P^{n}\right), \mathbf{k}\right) .
$$

Then by Theorem 3.6, $b^{r}\left(\mathcal{Z}_{P}\right)=\sum_{2 j-i=r} b^{-i, 2 j}$. It is easy to check that

$$
b^{0,0}=1, \quad b^{-q, 2 s}=0 \quad \text { if } \quad 0<s \leq q .
$$

Now, one can define Euler characteristics $\chi_{s}$ as

$$
\chi_{s}=\sum_{q=0}^{m}(-1)^{q} b^{-q, 2 s}, \quad s=0, \ldots, m
$$

and then define the series $\chi(t)$ as

$$
\chi(t)=\sum_{s=0}^{m} \chi_{s} t^{2 s}
$$

It can be shown that for this series the following identity holds

$$
\chi(t)=\left(1-t^{2}\right)^{m-n}\left(h_{0}+h_{1} t \ldots+h_{n} t^{2 n}\right) .
$$

This formula allows to express the $h$-vector $\left(h_{0}, \ldots, h_{n}\right)$ of a simple polytope $P^{n}$ in terms of the bigraded Betti numbers $b^{-q, 2 r}\left(\mathcal{Z}_{P}\right)$ of the corresponding manifold $\mathcal{Z}_{P}$.

At the end let us mention two more connections with well-known combinatorial results. Firstly, consider the first non-trivial MacMullen inequality for simple $P^{n}$ (see $[\mathbf{B r}])$ :

$$
h_{1} \leq h_{2}, \quad \text { for } \quad n \geq 3 .
$$

Using identity (4.5), one can express the above inequality in terms of the bigraded Betti numbers $b^{-q, 2 r}$ as follows:

$$
b^{3}\left(\mathcal{Z}_{P}\right)=b^{-1,4}\left(\mathcal{Z}_{P}\right) \leq\left(\begin{array}{c}
m-n \\
2
\end{array}\right), \quad \text { for } n \geq 3 .
$$

Secondly, let us consider the well-known Upper Bound for the number of faces of simple polytope. In terms of the $h$-vector it is as follows:

$$
h_{i} \leq\left(\begin{array}{c}
m-n+i-1 \\
i
\end{array}\right)
$$

(see $[\mathbf{B r}])$. Using the identity

$$
\left(\frac{1}{1-t^{2}}\right)^{m-n}=\sum_{i=0}^{\infty}\left(\begin{array}{c}
m-n+i-1 \\
i
\end{array}\right) t^{2 i},
$$

and formula (4.5), we deduce that the Upper Bound is equivalent to the following inequality:

$$
\chi(t) \leq 1, \quad|t| \leq 1 .
$$


It would be interesting to obtain a purely topological proof of inequalities (4.6) and (4.7).

\section{Concluding remarks}

As we mentioned in the introduction, the confluence of ideas from topology and combinatorics that gives rise to our notion of manifolds defined by simple polytopes ascends to geometry of toric varieties. Toric geometry enriched combinatorics of polytopes by very powerful topological and algebraic-geometrical methods, which led to solution of many well-known problems. Here we mention only two aspects. The first one is counting lattice points: starting from [Da] the Riemann-Roch theorem for toric varieties and related results were used for calculating the number of lattice points inside integral polytopes (see also $[\mathbf{F u}]$ ). The second aspect is the famous Stanley theorem [St1] that proves the necessity of MacMullen's conjecture for the number of faces of a simple (or simplicial) polytope. The proof uses the projective toric variety constructed from a simple polytope with vertices in integral lattice. This toric variety is not determined by the combinatorial type of the polytope: it depends also on integral coordinates of vertices. Many combinatorial types can be realized as integral simple polytopes in such a way that the resulting toric variety is smooth; in this case the Dehn-Sommerville equations follow from the Poncaré duality for ordinary cohomology, while the MacMullen inequalities follow from the Hard Lefschetz theorem. However, there are combinatorial types of simple polytopes that do not admit any smooth toric variety. The simplest examples are duals to the so-called cyclic polytopes of dimension $\geq 4$ with sufficiently many vertices (see [DJ, Corollary 1.23]). For such polytopes Stanley's proof uses the Poincaré duality and the Hard Lefschetz theorem for intersection cohomology of the corresponding (singular) toric variety. We mention that the Hard Lefschetz theorem for intersection cohomology is a very deep algebraic-geometrical result. Nevertheless, methods of toric geometry fail to give a proof of very natural generalization of MacMullen's conjecture to the case of simplicial spheres. The discussion of MacMullen's inequalities for simplicial spheres, Gorenstein complexes and related topics can be found in [St2]. On the other hand, our approach provides an interpretation of Dehn-Sommerville equations in terms of Poincaré duality in ordinary cohomology for any combinatorial simple polytope and gives a topological interpretation of MacMullen's inequalities. Moreover, our methods extend naturally to simplicial spheres. In can be easily seen that the construction of manifold $\mathcal{Z}_{P}$ and other constructions from our paper are equally applicable for non-polytopal simplicial spheres. Actually, an analog of $\mathcal{Z}_{P}$ can be constructed for any simplicial complex. In general case this fails to be a manifold, however it still decomposes into blocks of type $\left(D^{2}\right)^{q} \times T^{m-q}$ as described in the proof of Theorem 1.6. We call this space the moment-angle complex defined by simplicial complex. As in the case of a simple polytope, the moment-angle complex is homotopically equivalent to a certain coordinate subspace arrangement determined by the simplicial complex (see Section 2). In our paper [BP3] we study topology of moment-angle complexes and calculate the cohomology rings of general coordinate subspace arrangements.

\section{References}

[Au] M. Audin, The Topology of Torus Actions on Symplectic Manifolds, Progress in Mathematics 93, Birkhäuser, Boston Basel Berlin, 1991. 
[Co] D. A. Cox, Recent developments in toric geometry, in: Algebraic geometry (Proceedings of the Summer Research Institute, Santa Cruz, CA, USA, July 9-29, 1995), J. Kollar, (ed.) et al. Providence, RI: American Mathematical Society. Proc. Symp. Pure Math. 62 (pt.2), 389-436 (1997).

[Ba] V.V. Batyrev, Quantum Cohomology Rings of Toric Manifolds, Journées de Géométrie Algébrique d'Orsay (Juillet 1992), Astérisque 218, Sociéte Mathématique de France, Paris, 1993, pp. 9-34.

[Br] A. Brønsted, An introduction to convex polytopes, Springer-Verlag, New-York, 1983.

[BP1] V.M. Bukhshtaber and T.E. Panov, Algebraic topology of manifolds defined by simple polytopes (Russian), Uspekhi Mat. Nauk 53 (1998), no. 3, 195-196; English transl. in: Russian Math. Surveys 53 (1998), no. 3, 623-625.

[BP2] V. M. Buchstaber and T. E. Panov, Torus actions and combinatorics of polytopes (Russian), Trudy Matematicheskogo Instituta im. Steklova 225 (1999), 96-131; English transl. in: Proceedings of the Steklov Institute of Mathematics 225 (1999), 87-120; available at http://xxx.lanl.gov/abs/math.AT/9909166.

[BP3] V.M. Buchstaber and T.E. Panov, Torus actions, equivariant moment-angle complexes and coordinate subspace arrangements (Russian), Zap. Nauchn. Semin. Leningr. Otd. Mat. Inst. Steklova 266 (2000); available at http://xxx.lanl.gov/abs/math.AT/9912199.

[BR] V.M. Bukhshtaber and N. Ray, Toric manifolds and complex cobordisms (Russian), Uspekhi Mat. Nauk 53 (1998), no. 2, 139-140; English transl. in: Russian Math. Surveys 53 (1998), no. 2, 371-373.

[Da] V. Danilov, The geometry of toric varieties, (Russian), Uspekhi Mat. Nauk 33 (1978), no. 2, 85-134; English transl. in: Russian Math. Surveys 33 (1978), 97-154.

[DJ] M. Davis and T. Januszkiewicz, Convex polytopes, Coxeter orbifolds and torus actions, Duke Math. Journal 62, (1991), no. 2, 417-451.

[Fu] W. Fulton, Introduction to Toric Varieties, Princeton Univ. Press, Princeton, NJ, 1993.

[Ma] S. Maclane, Homology, Springer-Verlag, Berlin, 1963.

[Sm] L. Smith, Homological Algebra and the Eilenberg-Moore Spectral Sequence, Transactions of American Math. Soc. 129 (1967), 58-93.

[St1] R. Stanley, The number of faces of a convex simplicial polytope, Adv. in Math. 35 (1980), 236-238.

[St2] R. Stanley, Combinatorics and Commutative Algebra, Progress in Mathematics 41, Birkhauser, Boston, 1983.

[Zi] G. Ziegler, Lectures on Polytopes, Graduate Texts in Math. 152, Springer-Verlag, Berlin Heidelberg New-York, 1995.

Department of Mathematics and Mechanics, Moscow State University, 119899 Moscow, RUSSIA

E-mail address: buchstab@mech.math.msu.su

E-mail address: tpanov@mech.math.msu.su 